\title{
Cardiovascular Disorder
}

National Cancer Institute

\section{Source}

National Cancer Institute. Cardiovascular Disorder. NCI Thesaurus. Code C2931.

A non-neoplastic or neoplastic disorder affecting the heart or the vessels (arteries, veins and lymph vessels). Representative examples of non-neoplastic cardiovascular disorders are endocarditis and hypertension. Representative examples of neoplastic cardiovascular disorders are endocardial myxoma and angiosarcoma. 